\title{
Uma Análise sobre o Desempenho de Alunos de Graduação em Disciplinas Iniciais de Programação
}

\author{
Erivan Lima $^{1,2}$, Emanuel F. Coutinho ${ }^{3}$ \\ ${ }^{1}$ Instituto Universidade Virtual (UFC Virtual) \\ ${ }^{2}$ Universidade Federal do Ceará (UFC) - Fortaleza, CE - Brasil \\ ${ }^{3}$ Universidade Federal do Ceará (UFC) - Quixadá, Brasil \\ erivan@alu.ufc.br, emanuel.coutinho@ufc.br
}

\begin{abstract}
Teaching programming is a complex task that requires a lot of effort by teachers, especially when the theory should be aligned with practice. There are several difficulties encountered by teachers and students during programming disciplines, culminating in high failure and dropout rates. This work aims to analyze the academic performance of students in an initial discipline of programming, in an undergraduate course. The methodology of this article was subdivided into four steps: data collection, data processing, hypothesis creation and analysis, through visualization of data in the form of Venn diagrams, in a computational tool. Visualization of the data enabled a simple analysis of academic information (scores, attendance and school failure), allowing identification of situations and support for decision making.
\end{abstract}

Resumo. $O$ ensino de programação é uma tarefa complexa que requer muito esforço por parte dos docentes, especialmente quando a teoria deve ser alinhada com a prática. São diversas as dificuldades encontradas por docentes e discentes durante as disciplinas de programação, culminando em altas taxas de reprovação e evasão. Este trabalho tem como objetivo analisar o desempenho acadêmico dos alunos em uma disciplina inicial de programação de um curso de graduação. A metodologia deste artigo se subdividiu em quatro etapas: coleta de dados, tratamento de dados, criação de hipóteses e análise das mesmas, por meio de visualização de dados em forma de diagramas de Venn, em uma ferramenta computacional. A visualização dos dados possibilitou uma análise simples de informações acadêmicas (notas, frequência e reprovação), permitindo identificação de situações e apoio para tomada de decisão.

\section{Introdução}

O ensino de qualquer disciplina em áreas técnicas constitui um conjunto de desafios para o docente, tanto pelo constante surgimento de novas tecnologias e ferramentas, como pela busca por estratégias na tentativa de se alinhar às novas práticas de aquisição de conhecimento adotadas pelos aprendizes atuais [Coutinho et al. 2018b]. Em particular, o ensino de lógica de programação e consequentemente a codificação são tarefas complexas que requerem muito esforço por parte dos docentes. Além disso, um desafio é auxiliar o aluno a associar a teoria ministrada com a prática, pois os alunos geralmente ingressam nos cursos de graduação com os mais variados perfis. 
VIII Congresso Brasileiro de Informática na Educação (CBIE 2019)

Anais do XXX Simpósio Brasileiro de Informática na Educação (SBIE 2019)

Disciplinas com a intenção de adequar teoria com prática são normalmente muito difíceis de se conduzir devido à necessidade de se tratar fatores técnicos, como linguagens de programação e ferramentas, e fatores humanos como comunicação e gestão, tudo ao mesmo tempo [Coutinho et al. 2016]. Coletar dados sobre o desempenho dos discentes e docentes não é trivial, pois existem muitas variáveis envolvidas, tais como metodologia, didática e tecnologias [Coutinho et al. 2018b].

Cada vez mais os cursos superiores agregam em seus currículos disciplinas de programação [de Souza e Coelho 2015][Coutinho et al. 2017]. Contudo, para a aprendizagem das disciplinas iniciais de programação é necessário algumas habilidades por parte do aprendiz, como o raciocínio lógico-matemático [de Jesus e Brito 2009]. Muitas vezes, alunos durante as disciplinas iniciais de programação enfrentam dificuldades na aquisição do conhecimento, ocasionando altas taxas de reprovação e evasão [de Farias et al. 2018].

Com o advento tecnológico, as instituições educacionais fazem crescentemente uso de Ambientes Virtuais de Aprendizagem (AVAs), que permite tanto o avanço da Educação à Distância quanto do ensino presencial, fazendo com que essas instituições disponham cada vez mais uma grande quantidade de dados acadêmicos [Maschio et al. 2018]. Dentre esses dados estão aqueles relacionados ao desempenho dos alunos durante as disciplinas, como por exemplo notas, frequências, aprovações ou reprovações. Porém, muitas vezes eles se encontram em diversos locais e com diferentes formatos, e quando devidamente tratados e analisados podem se transformar em importantes conhecimentos acerca de turmas e alunos [Cavalcanti et al. 2014].

Neste cenário, onde muitas vezes as disciplinas iniciais de programação apresentam um alto índice de reprovação e evasão de alunos, este trabalho tem como objetivo analisar o desempenho dos alunos em uma disciplina inicial de programação de um curso de graduação. Para atingir esse objetivo, utilizou-se uma aplicação para uma visualização de dados acadêmicos extraídos do sistema de gestão acadêmica utilizado no curso.

Este trabalho está dividido nas seguintes seções, além desta introdução: a Seção 2 apresenta alguns trabalhos relacionados; na Seção 3 a metodologia do trabalho é descrita; a Seção 4 apresenta a ferramenta desenvolvida; na Seção 5 os resultados e análises do estudo são descritos; e por fim, a seção 6 apresenta a conclusão e trabalhos futuros.

\section{Trabalhos Relacionados}

Diversos trabalhos na literatura analisam e discutem dados oriundos de disciplinas de programação com o objetivo de adquirir conhecimentos úteis para o aprimoramento, redução das altas taxas de evasão e reprovação nessas disciplinas.

Oliveira et al. (2017) discutiram os problemas que os discentes percebem durante o curso sobre diversos aspectos, além das relações entre a qualidade da metodologia, grade curricular, estrutura do curso e satisfação dos alunos, fazendo uso de dados coletados por meio de questionários de pesquisas com os discentes nos anos de 2016 à 2017.

Coutinho et al. (2017) apresentaram e analisaram experiências de uma disciplina inicial de programação na graduação em cinco turmas. Verificou-se o desempenho dos discentes por meio de estatística descritiva, com média, mediana, valores mínimo e máximo, desvio padrão da média final, quantidade de faltas dos alunos e situação (variando entre aprovado, reprovado ou trancado) dos alunos ao final da disciplina. 
VIII Congresso Brasileiro de Informática na Educação (CBIE 2019)

Anais do XXX Simpósio Brasileiro de Informática na Educação (SBIE 2019)

A evasão escolar é uma crescente dificuldade enfrentada em diversas áreas do conhecimento, sendo um problema que deve ser tratado com cuidado [Coutinho et al. 2018a]. Nesse trabalho, visualizações de dados acadêmicos foram geradas para se estudar problemas de maneira geral em um curso de graduação, e assim se identificar causas para evasão.

Ribeiro et al. (2018) realizaram análises quantitativas do desempenho de dois grupos de discentes, com o primeiro formado por alunos que utilizaram a ferramenta $\mathrm{Co}$ deBench sem gamificação, e o segundo por alunos que fizeram uso da mesma ferramenta com elementos de gamificação, mostrando a evolução no desempenho dos discentes.

Guedes (2014) avaliaram o desempenho dos estudantes de Engenharias em uma disciplina inicial de programação a fim de identificar possíveis explicações para os altos índices de reprovação e desistências reportados naquela instituição. $\mathrm{O}$ estudo foi realizado em um semestre acadêmico de 2014 com 147 estudantes divididos em quatro turmas, das quais uma era composta em sua maioria de alunos novatos e as outras três de alunos reprovados. Os dados utilizados neste estudo foram coletados por meio de questionários.

Schorr e Bercht (2018) analisaram as médias dos três últimos anos do Ensino Médio de 137 alunos e seus respectivos desempenhos em uma disciplina de programação numa IES, para investigar as disciplinas que podem contribuir para o desenvolvimento das competências e habilidades necessárias para a aprendizagem de Algoritmos e Programação no Ensino Superior.

A Tabela 1 apresenta alguns critérios de comparação entre os artigos.

Tabela 1. Comparação entre trabalhos relacionados (Ferramenta - utilização ou
construção de ferramentas, Visualizações - utilização de visualização de dados,
e Experimentos - realização de experimentos)
\begin{tabular}{l|c|c|c}
\hline Trabalho & Ferramenta & Visualizações & Experimentos \\
\hline [Oliveira et al. 2017] & $\operatorname{sim}$ & $\operatorname{sim}$ & sim \\
\hline [Coutinho et al. 2017] & não & sim & não \\
\hline [Coutinho et al. 2018a] & não & sim & sim \\
\hline [Ribeiro et al. 2018] & não & sim & sim \\
\hline [Guedes 2014] & não & sim & sim \\
\hline [Schorr e Bercht 2018] & não & sim & sim \\
\hline
\end{tabular}

\section{Metodologia}

A metodologia deste trabalho é composta por quatro passos: coleta de dados, tratamento de dados, formulação de questões de pesquisas e a análise dos dados.

\subsection{Coleta de dados}

O curso de graduação a ter as turmas de programação iniciais analisadas é o curso de Sistemas e Mídias Digitais, da Universidade Federal do Ceará. A disciplina a qual teve as turmas envolvidas na pesquisa foi a disciplina de Programação I.

O processo de coleta de dados se deu pela extração de dados do Sistema Integrado de Gestão de Atividades Acadêmicas (SIGAA), sistema de gestão acadêmica utilizado pela universidade, onde foi possível adquirir as notas, as frequências, os registros de aprovação e a lista de alunos que realizaram a avaliação institucional durante a disciplina, do total de 113 alunos de quatro turmas de uma disciplina inicial de programação. 
VIII Congresso Brasileiro de Informática na Educação (CBIE 2019)

Anais do XXX Simpósio Brasileiro de Informática na Educação (SBIE 2019)

\subsection{Tratamento de dados}

Inicialmente os dados encontravam-se em diferentes formatos. As notas, frequências e os registros de aprovação estavam registrados em PDF e os dados de quais alunos realizaram a avaliação institucional estavam em arquivos CSV (Comma-separated values). Para seu tratamento, os dados foram transferidos para uma planilha do Google Spreadsheets, facilitando assim sua organização, fazendo-se uso de tabelas, em que cada linha foram colocados os dados referentes a cada aluno da amostra.

\subsection{Formulação de questões de pesquisa}

Perante este trabalho, algumas hipóteses relacionadas ao desempenho dos alunos em disciplinas de programação foram formuladas.

A primeira hipótese a ser analisada é "Assiduidade dos alunos interfere diretamente em suas notas". Essa hipótese parte do pressuposto de que para um aluno ter um bom desempenho, realizando boas avaliações e conseguindo boas notas, é necessário que esse aluno compareça as aulas e apresente uma boa assiduidade durante a disciplina.

A Universidade Federal do Ceará, assim como muitas instituições de ensino superior, conta com uma avaliação institucional, que é um formulário realizado ao final dos semestres, formado de questões relacionadas à metodologia dos professores e das disciplinas cursadas, com intuito de prover informações que auxiliem no aprimoramento de seus professores e de suas disciplinas. Entretanto, muitos alunos podem se sentir desmotivados a avaliar essas disciplinas. Pensando nisso, surgiu outra hipótese, que é "Alunos aprovados se sentem mais motivados a avaliar a disciplina que alunos reprovados".

A terceira hipótese a ser analisada é "Alunos com boa assiduidade e com nota acima da média tendem a avaliar a disciplina mais que alunos com baixa assiduidade e com nota inferior a média". Partindo da mesma ideia da segunda hipótese, mas focando em grupos mais fechados, e alterando as variáveis observadas, agora tratando não mais com aprovados e reprovados, e sim com as questões da assiduidade e das notas apresentadas pelos alunos ao final da disciplina.

\subsection{Análise dos Dados}

De posse dos dados acadêmicos dos alunos, e uma vez estes portados para serem lidos na ferramenta, cada uma das hipóteses teria um ou mais gráficos que as representasse. Assim, seria possível analisar cada hipótese e relacionar os dados entre si, possibilitando a identificação de situações que confirmasse ou não as hipóteses.

\section{Ferramenta}

A FADA $^{1}$ (Ferramenta para Análise de Dados Acadêmicos) dispõe de um mecanismo de seleção das variáveis previamente estabelecidas (notas, frequências e situação na disciplina). A ferramenta permite a visualização das relações lógicas entre uma coleção de conjuntos, onde cada conjunto é uma coleção de dados que possui algo em comum, e nos círculos que se sobrepõem acontece a interseção, o local onde se encontra os dados com qualidades em comum, através da utilização de diagramas de Venn. Uma visão geral da ferramenta está exibida na Figura 1.

\footnotetext{
${ }^{1}$ https://erivanufc.github.io/diagrama/index.html
} 
VIII Congresso Brasileiro de Informática na Educação (CBIE 2019)

Anais do XXX Simpósio Brasileiro de Informática na Educação (SBIE 2019)

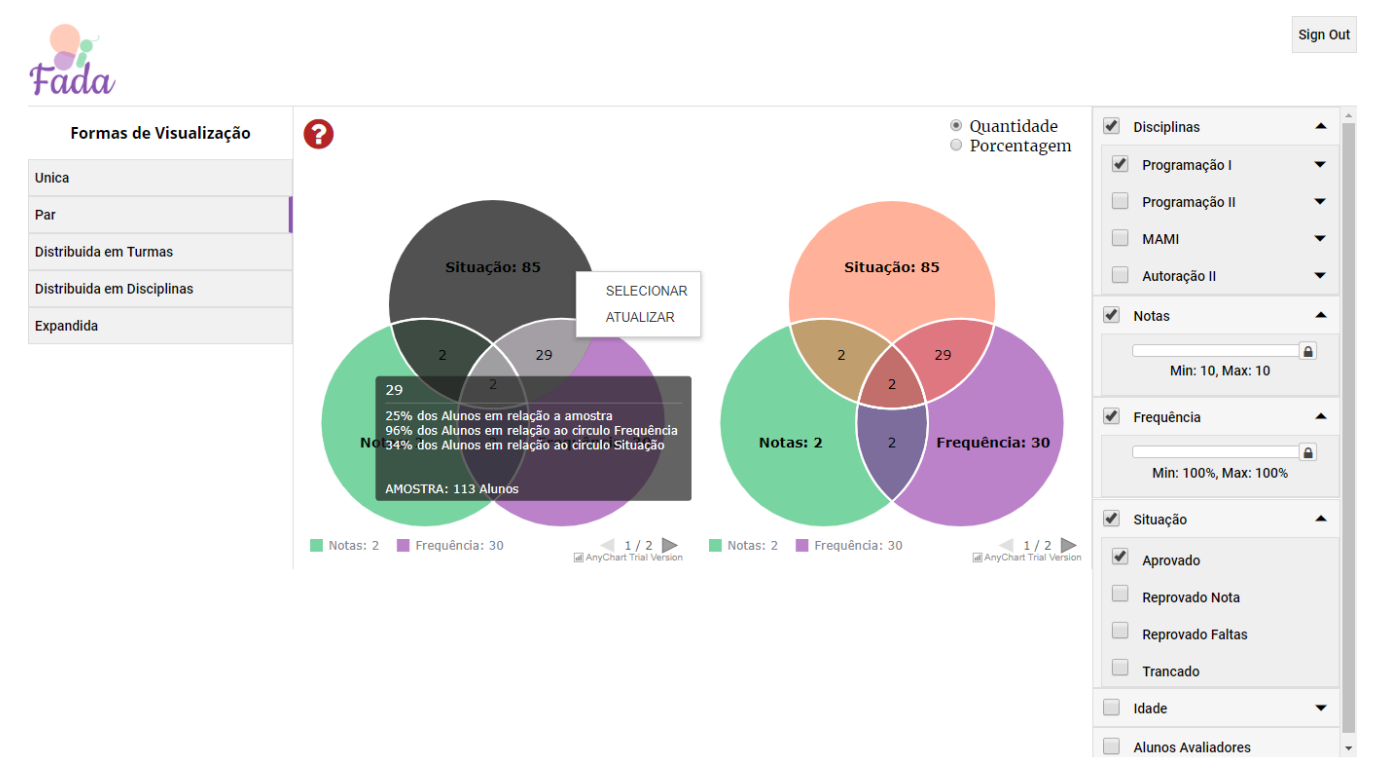

Figura 1. Visão geral da Ferramenta para Análise de Dados Acadêmicos

A aplicação consiste de cinco módulos de visualização, que são: única, par, distribuída em turmas, distribuída em disciplinas e a expandida. O modulo "única" permite visualizar apenas um diagrama. A opção "par" possibilita que dois diagramas sejam mostrados ao mesmo tempo. Tanto a opção "distribuída em turmas" como a opção "distribuída em disciplinas" possibilitam ver uma evolução ou uma regressão por parte do desempenho dos alunos, seja dispondo os dados referentes a determinadas turmas de uma disciplina, ou visualizando as disciplinas, onde os dados são divididos em conjuntos formados pelos dados de cada disciplina. Já a visualização "expandida" permite que após criado um diagrama, os dados sejam reutilizados um novo diagrama com valores diferentes.

\section{Resultados e Análises}

A Tabela 2 exibe dados da situação dos alunos para cada turma analisada, discriminados por quantidade de alunos, quantidade de aprovações, quantidade de reprovações, nota e frequência média da turma, total de alunos avaliadores, e a média de cada coluna.

Tabela 2. Situação dos alunos por turma e total (Total de alunos (T), Total de alu-
nos aprovados (Apr), Total de alunos reprovados por nota (RepN), Total de alu-
nos reprovados por frequência (RepF), Nota média da turma (NotaM), Frequência
média da turma (FreqM), Total de alunos avaliadores (TAv))
\begin{tabular}{c|c|c|c|c|c|c|c}
\hline Turma/Semestre & T & Apr & RepN & RepF & NotaM & FreqM & TAv \\
\hline Turma 2017.1 (2A) & 29 & 18 & 8 & 3 & 6.05 & $91.9 \%$ & 22 \\
\hline Turma 2017.1 (3A) & 27 & 17 & 9 & 1 & 5.63 & $90.3 \%$ & 19 \\
\hline Turma 2018.1 (1A) & 28 & 24 & 3 & 1 & 6.91 & $93.1 \%$ & 14 \\
\hline Turma 2018.1 (2A) & 29 & 26 & 2 & 1 & 7.65 & $91.1 \%$ & 14 \\
\hline \hline MÉDIA & 28.25 & 21.25 & 5.50 & 1.50 & 6.56 & $91.6 \%$ & 17.25 \\
\hline
\end{tabular}

O total de alunos que estavam matriculados nas turmas foi 113 . Não ocorreram trancamentos, então todos ou foram aprovados ou foram reprovados. A quantidade de alunos aprovados foi 85 . Já o número de reprovados foi de 28 , divididos em 22 reprovados 
VIII Congresso Brasileiro de Informática na Educação (CBIE 2019)

Anais do XXX Simpósio Brasileiro de Informática na Educação (SBIE 2019)

por nota e 6 reprovados por faltas. Após os dados serem portados para a ferramenta, a tarefa foi gerar visualizações para a verificação das hipóteses.

\subsection{A assiduidade dos alunos interfere diretamente em suas notas}

Foram criados dois diagramas (Figura 2), formados a partir das variáveis "frequência", "notas" e "situação". Ambos diagramas tiveram como valor mínimo 90\% para a variável "frequência" e valor máximo 100\%. Já o intervalo definido para a variável "notas" no diagrama à esquerda tem como valor mínimo 9 e máximo 10, enquanto que no diagrama à direita a margem inicia com valor 7 e termina com valor 10 . Na variável "situação", no diagrama à esquerda, foram selecionados os valores "reprovados por nota" e "reprovados por faltas", enquanto que no diagrama à direita foram selecionados os "aprovados".
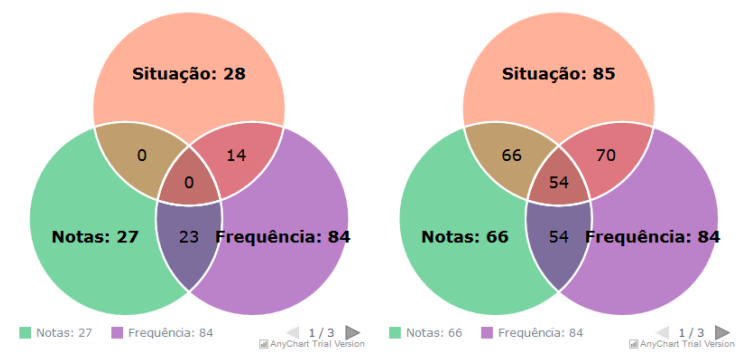

Figura 2. Diagrama à esquerda formado pela quantidade de alunos com nota igual ou maior que 9 (verde), quantidade de alunos com frequência igual ou acima de $90 \%$ (lilás) e a quantidade de alunos reprovados (salmão). Diagrama à direita formado pela quantidade de alunos com nota igual ou maior que 7 (verde), quantidade de alunos com frequência igual ou acima de $90 \%$ (lilás) e a quantidade de alunos aprovados (salmão)

Logo é possível observar, de acordo com o diagrama à esquerda da Figura 2, que dos 84 alunos que obtiveram frequência igual ou maior que 90\%, 23 deles adquiriram uma nota igual ou maior que 9 , representando assim $27.38 \%$ dos 84 alunos, quase $1 / 3$ do conjunto, apresentando ser um valor baixo. Entretanto, observando o diagrama do lado direito da Figura 2 é possível observar que desses mesmos 84 alunos, 54 obtiveram nota igual ou superior a 7, sendo aprovados na disciplina. Esse grupo formado por alunos com frequência igual ou acima de $90 \%$ e nota igual ou superior a 7 equivalem à $81.82 \%$ de todos os alunos aprovados na disciplina com nota superior ou igual a média, que é representado no diagrama pelos 66 alunos na interseção entre "situação" e "notas", ou seja, $81.82 \%$ de todos os alunos aprovados com nota superior ou igual a média frequentaram $90 \%$ da disciplina ou mais. Além de que esse grupo de alunos possui uma média das notas de 8.53, quase 2 pontos a mais que os 6.56 da média geral das notas presente na Tabela 2.

A Tabela 2 reforça alguns pontos positivos. Pois mesmo que a frequência média entre as turmas sejam muito próximas, é visto que a turma com menor média das frequências é justamente a que apresenta menor média das notas e a turma que apresenta maior média das frequências é a segunda turma dentre as quatro da amostra, na média das notas. Entretanto a média das frequências em todas as turmas é em torno de 90\%, 57.6 em valores normais, o que significa uma média de 6.4 faltas, uma quantidade podendo ser considerada baixa, porém apenas uma turma conseguiu alcançar uma média das notas superior a média para aprovação. 
VIII Congresso Brasileiro de Informática na Educação (CBIE 2019)

Anais do XXX Simpósio Brasileiro de Informática na Educação (SBIE 2019)

\subsection{Os alunos aprovados se sentem mais motivados a avaliar a disciplina que alunos reprovados}

Foram criados dois diagramas (Figura 3), os quais foram formados a partir das variáveis "situação", "avaliadores" e "frequência". Para o diagrama à esquerda foi selecionado o valor "aprovados" para a variável "situação", enquanto que no diagrama à direita foram selecionados os valores "reprovados por nota" e "reprovados por faltas". Para a criação de ambos diagramas a variável "avaliadores" foi selecionada.
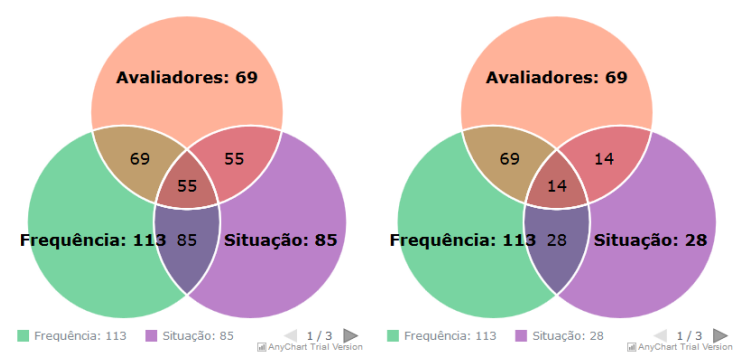

Figura 3. Diagrama à esquerda formado pelo tamanho da amostra (verde), quantidade de alunos aprovados (lilás) e a quantidade de alunos que avaliaram a disciplina (salmão). Diagrama à direita formado pelo tamanho da amostra (verde), quantidade de alunos reprovados (lilás) e a quantidade de alunos que avaliaram a disciplina (salmão)

Dos 85 alunos aprovados, 55 participaram da avaliação institucional, ou seja, $64.70 \%$ dos aprovados avaliaram a disciplina, enquanto que dos 28 alunos reprovados, seja por faltas ou por nota, 14 alunos avaliaram a disciplina. Ou seja $50 \%$ dos reprovados avaliaram a disciplina de Programação I, o que significa que de acordo com a amostra utilizada houve mais participação na avaliação institucional dentro do grupo de alunos aprovados do que no grupo de alunos reprovados.

\subsection{Alunos com boa assiduidade e com nota acima da média tendem a avaliar a disciplina mais que alunos com baixa assiduidade e com nota inferior à média}

Para esta hipótese "boa assiduidade" foi considerada como uma frequência igual ou superior a $75 \%$, o necessário para que o aluno não seja reprovado por faltas. Foram criados dois diagramas, ambos sendo formados a partir das variáveis "notas", "avaliadores" e "situação". As margens definidas para variável "notas" do diagrama à esquerda foram inicialmente de 7, que é equivalente a média, e igual ou inferior a 10, enquanto que no diagrama à direita, as margens possuem como valor inicial igual a 0 e valores menores que 7. Para a variável "situação", no diagrama à esquerda foi selecionado o valor "aprovados", enquanto que o diagrama à direita está "reprovados por faltas" como valor selecionado. Nessa análise, mesmo a hipótese utilizando os termos "boa assiduidade" e "baixa assiduidade", esses diagramas não utilizaram de dados de frequência, preferindo-se os dados dos alunos reprovados por faltas.

Para esclarecer a diferença entre esta hipótese e a segunda, é importante destacar que no grupo formado por alunos com boa assiduidade e com nota acima da média, em comparação com o grupo de alunos aprovados, são excluídos aqueles alunos que obtiveram nota inferior à média mas foram aprovados após realizarem uma avaliação final. 

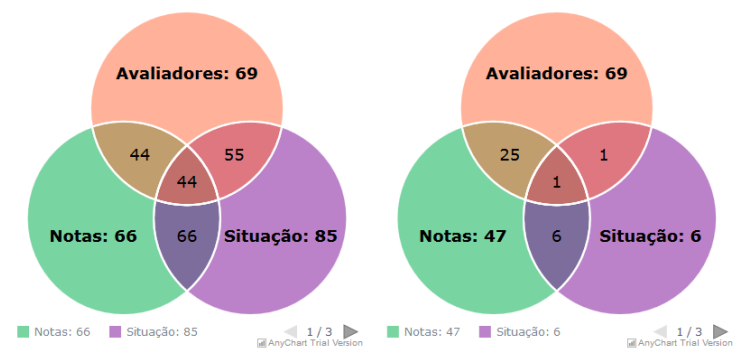

Figura 4. Diagrama à esquerda formado pela quantidade de alunos com nota igual ou superior à média (verde), quantidade de alunos aprovados (lilás) e a quantidade de alunos que avaliaram a disciplina (salmão). Diagrama à direita formado pela quantidade de alunos com nota igual ou inferior à média (verde), quantidade de alunos reprovados por faltas (lilás) e a quantidade de alunos que avaliaram a disciplina (salmão)

Na Figura 4 podemos observar que dos 113 alunos pertencentes a amostra, 66 deles conseguiram terminar a disciplina de Programação I com nota igual ou maior que 7 e que também foram aprovados, sendo indicado pela interseção entre as variáveis "notas" e "situação", o que indica que não tiveram uma baixa assiduidade, pois não foram reprovados por faltas. Destes 66 alunos, 44 fizeram a avaliação institucional, ou seja, aproximadamente $66 \%$ desse grupo, enquanto que dos 6 alunos reprovados por faltas que tiveram nota menor que a média, apenas 1 aluno respondeu a avaliação institucional, o que significa que $16 \%$ desse grupo respondeu a avaliação institucional.

\subsection{Análise dos Resultados}

Nos resultados da primeira hipótese é reforçado que quanto maior a frequência de um aluno maior suas chances de adquirir boas notas, e o inverso também é visto, quanto menor a frequência maiores as chances de adquirir notas ruins. Entretanto ao observarmos a Tabela 2, percebemos que mesmo as turmas tendo uma frequência média muito alta, a média de suas notas ainda é baixa, o que pode ser causado devido ao fato dos alunos em geral serem assíduos, mas o número de alunos com notas boas e notas ruins serem próximos, e quando visto a média, os valores variam um pouco abaixo da média.

É visto nos resultados da segunda hipótese que o grupo formado pelos alunos com boa frequência e nota, e os alunos com nota inferior à média mas aprovados após realizarem uma avaliação final, é o grupo de alunos que mais tende a avaliar a disciplina em relação aos reprovados. Esses resultados mostram um aspecto negativo nessas edições da avaliação institucional, que foi o caso dos alunos com baixo desempenho não comunicarem através da avaliação institucional motivos da sua possível insatisfação com a disciplina ou motivos gerais de apresentarem baixa assiduidade durante a disciplina.

Através desses resultados podemos notar um baixo rendimento dos alunos, principalmente através dos resultados da primeira hipótese, onde foi possível perceber que dos 84 alunos que obtiveram frequência igual ou acima de $90 \%$, apenas 23 tiveram nota igual ou acima de 9. Mesmo que a assiduidade interfira em sua nota, com esse dado é possível perceber que apenas uma boa assiduidade dos alunos não é suficiente para que consigam notas exemplares, o que é ainda reforçado pela Tabela 2, onde nenhuma das turmas conseguiu uma média das notas superior a 8 , mesmo que as médias das frequências em todas 
VIII Congresso Brasileiro de Informática na Educação (CBIE 2019)

Anais do XXX Simpósio Brasileiro de Informática na Educação (SBIE 2019)

as turmas sejam maiores que $90 \%$.

O número de alunos que avaliam a disciplina está abaixo do esperado, visto que ao observarmos a Tabela 2, é possível notar que a média dos alunos na disciplina é 28.25, e a média de alunos que avaliaram a avaliação institucional é 17.25 , o que equivale a $61.06 \%$. Os resultados das hipóteses 2 e 3 reforçaram ainda mais a ideia que alunos com baixo desempenho tendem a avaliar menos que alunos com melhores desempenhos. Essa situação propulsiona uma dificuldade maior em adquirir informações através da avaliação institucional que auxiliem no desenvolvimento da disciplina.

Possivelmente o motivo de alunos com melhores desempenhos avaliarem a disciplina mais que alunos com desempenhos inferiores se dá ao fato de que alunos com bons desempenhos terminam a disciplinas com uma satisfação maior e uma sensação de dever cumprido, sendo assim motivados a avaliar a disciplina, enquanto que alunos com desempenhos inferiores sentem uma insatisfação e um aborrecimento com a disciplina, sentido-se menos motivados a avaliar.

\section{Conclusões e Trabalhos Futuros}

Este trabalho analisou dados coletados de turmas de uma disciplina de programação para alunos iniciantes em um curso de graduação, realizado por meio de visualizações de dados acadêmicos obtidos a partir da ferramenta de controle acadêmico da instituição.

Uma dificuldade presente foi a obtenção dos dados. Muitas vezes existem ferramentas computacionais, como os sistemas de gerenciamento acadêmicos das instituições, com diversos dados disponíveis. Porém, o acesso a esses dados é limitado, por questões de controle de acesso, segurança e sigilo. Geralmente esses dados estão em formatos variados, requerendo um tratamento para deixá-los compatíveis com outras ferramentas.

Este trabalho reforçou a ideia que a frequência influencia nas notas dos alunos. Notou-se que mesmo os alunos apresentando uma assiduidade muita alta, percebido pela frequência média das turmas, eles não conseguiram transformar isso em boas notas. Com isso é necessário um estudo das participações dos alunos em aulas, verificando se essas aulas estão despertando o interesse dos alunos e se estão sendo capazes de repassarem conhecimentos aos alunos. Além disso, neste trabalho foi possível observar um número abaixo do esperado de alunos que realizaram a avaliação institucional, principalmente pelos alunos reprovados, tendo assim a necessidade de se incentivar mais os alunos a preencherem a avaliação institucional, indicando sua importância para as futuras turmas.

Dentre as contribuições que este trabalho deixa para futuras pesquisas está a ferramenta, que mostrou-se capaz de exercer sua função no auxílio para as hipóteses propostas. Como trabalhos futuros, tem-se a intenção de adicionar mais funcionalidades à ferramenta para suportar ainda mais a análise de dados de maneira visual. Outro trabalho a ser planejado é identificar causas para evasão e reprovação, e alinhar estratégias com professores da disciplina de programação e coordenações, reduzindo assim esses números.

\section{Agradecimentos}

Este trabalho foi realizado com recursos do projeto Aplicação de Técnicas de Visualização de Dados para Identificação de Problemas Relacionados a Lógica e Fundamentos de Programação, conforme Edital 02/18 (PIBIC 2018/2019), da Universidade Federal do Ceará (UFC). 
VIII Congresso Brasileiro de Informática na Educação (CBIE 2019)

Anais do XXX Simpósio Brasileiro de Informática na Educação (SBIE 2019)

\section{Referências}

Cavalcanti, A., dos Santos, N. N., e Cavalcanti, J. L. (2014). Mineração e visualização de dados educacionais: identificação de fatores que afetam a motivação de alunos na educação a distância. XIV Escola Regional de Computação Bahia, Alagoas e Sergipe (ERBASE).

Coutinho, E. F., Bezerra, J. D. H., Bezerra, C. I. M., e Moreira, L. O. (2018a). Uma análise da evasão em cursos de graduação apoiado por métricas e visualização de dados. In XXIV Workshop de Informática na Escola (WIE 2018).

Coutinho, E. F., Bonates, M. F., e Moreira, L. O. (2018b). Relato sobre o uso de uma ferramenta de desenvolvimento de jogos para o ensino introdutório de lógica de programação. In IV Workshop de Ensino em Pensamento Computacional, Algoritmos e Programação (WAlgProg).

Coutinho, E. F., de Lima, E. T., e Santos, C. C. (2017). Um panorama sobre o desempenho de uma disciplina inicial de programação em um curso de graduação. Revista Tecnologias na Educação, 19(9).

Coutinho, E. F., Gomes, G. A. M., e Leite, A. J. M. (2016). Applying design thinking in disciplines of systems development. In 2016 8th Euro American Conference on Telematics and Information Systems (EATIS), pages 1-8.

de Farias, C. M., Azevedo, F. P., e de Jesus Dias, J. E. (2018). Uma abordagem gamificada para o ensino de lógica de programação: relato de experiência. In $26^{\circ}$ Workshop sobre Educação em Computação (WEI 2018), volume 26, Porto Alegre, RS, Brasil. SBC.

de Jesus, A. e Brito, G. S. (2009). Concepção de ensino-aprendizagem de algoritmos e programação de computadores: a prática docente. Varia Scientia, 9(16):149-158.

de Souza, J. F. e Coelho, S. A. (2015). Uma biblioteca gráfica para aprendizagem de algoritmos e estruturas de dados. Revista Brasileira de Informática na Educação, 23(01): 110 .

Guedes, E. B. (2014). Um estudo observacional sobre a disciplina introdutória de programação. In Anais do XX Workshop de Informática na Escola (WIE 2014).

Maschio, P., Vieira, M. A., Costa, N., Melo, S. D., e Júnior, C. P. (2018). Um panorama acerca da mineração de dados educacionais no brasil. In Anais do XXIX Simpósio Brasileiro de Informática na Educação (SBIE 2018).

Oliveira, B. P., Balan, G. R., Soares, L. P., e Coutinho, E. F. (2017). Uma ferramenta para o auxílio do acompanhamento da qualidade e problemas de um curso de graduação. Revista Sistemas e Mídias Digitais (RSMD), 2(2).

Ribeiro, R. B., Fernandes, D., de Carvalho, L. S. G., e Oliveira, E. (2018). Gamificação de um sistema de juiz online para motivar alunos em disciplina de programação introdutória. In Anais do XXIX Simpósio Brasileiro de Informática na Educação (SBIE 2018).

Schorr, M. e Bercht, M. (2018). Análise longitudinal do desempenho dos estudantes de ensino médio e estudantes de nível superior para algoritmos e programação. In Anais dos Workshops do VII Congresso Brasileiro de Informática na Educação (CBIE 2018). 